\title{
OPTIMAL CONTROL PROBLEMS WITH NONREGULAR MIXED CONSTRAINTS 1
}

\author{
Maria do Rosário de Pinho* \\ Maria Margarida A. Ferreira* \\ Fernando A. C. C. Fontes ${ }^{* *}$ \\ * ISR and DEEC, Faculdade de Engenharia da Universidade do \\ Porto, Rua Dr. Roberto Frias, 4200-465 Porto, Portugal. \\ Fax: +351225081440, mrpinho@fe.up.pt, mmf@fe.up.pt \\ ** Officina Mathematica, \\ Departamento de Matemática para a Ciência e Tecnologia, \\ Universidade do Minho \\ 4800-058 Guimarães, Portugal. \\ fax: +351253510401 \\ ffontes@mct.uminho.pt
}

\begin{abstract}
In this paper we derive necessary conditions for a simple class of mixed constrained optimal control problems that do not satisfy known regularity conditions on the mixed constraints. Such problems are a particular class of nonregular problems. Notably the necessary conditions we derive are stated in terms of measures.
\end{abstract}

Keywords: Optimal control, mixed constraints, nonsmooth maximum principle.

\section{INTRODUCTION}

Consider the following mixed constrained optimal control problem:

$$
(P)\left\{\begin{array}{rlrl}
\text { Minimize } l(x(0), x(1)) & & \\
\text { subject to } & & \\
\dot{x}(t) & =f(t, x(t), u(t)) & & \text { ae } \\
0 & =b(t, x(t), u(t)) & & \text { ae } \\
0 & \geq g(t, x(t), u(t)) & & \text { ae } \\
(x(0), x(1)) & \in C & &
\end{array}\right.
$$

where $l: \mathbb{R}^{n} \times \mathbb{R}^{n} \rightarrow \mathbb{R}, C \subset \mathbb{R}^{n} \times \mathbb{R}^{n}$ and

$$
(f, b, g):[0,1] \times \mathbb{R}^{n} \times \mathbb{R}^{k} \rightarrow \mathbb{R}^{n} \times \mathbb{R}^{m_{b}} \times \mathbb{R}^{m_{g}} .
$$

For $(P)$ define the scalar valued function

$$
\begin{gathered}
H(t, x, u, p, q, r)= \\
p \cdot f(t, x, u)+q \cdot b(t, x, u)+r \cdot g(t, x, u) .
\end{gathered}
$$

\footnotetext{
1 Supported by FCT and FEDER, Project POSC/EEASRI/61831/2004 and ISR-P, Unit 147.
}

Take $(\bar{x}, \bar{u})$ to be a solution to $(P)$. With respect to this process the set $\mathcal{I}_{a}(t)$ denotes the set of indexes of the active constraints, i.e.,

$$
\begin{gathered}
\mathcal{I}_{a}(t)= \\
\left\{i \in\left\{1, \ldots, m_{g}\right\} \mid g_{i}(t, \bar{x}(t), \bar{u}(t))=0\right\},
\end{gathered}
$$

and $q_{a}(t)$ denotes the cardinality of $\mathcal{I}_{a}(t)$. Also

$$
\nabla_{u} g^{\mathcal{I}_{a}(t)}(t, \bar{x}(t), \bar{u}(t)) \in \mathbb{R}^{q_{a}(t) \times k},
$$

(if $q_{a}(t)=0$, then the latter holds vacuously) is the matrix we obtain after removing from $\nabla_{u} g(t, \bar{x}(t), \bar{u}(t))$ all the rows of index $i \notin \mathcal{I}_{a}(t)$.

Necessary conditions for smooth problem $(P)$ are well known (see, for example, (Hestenes, 1966; Neustadt, 1976; Schwarzkopf, 1976; Steffani and Zezza, 1996; Milyutin and Osmolovskii, 1998; Dmitruk, 1993; Arutyunov, 2000)). Maximum principles have also been generalized to cover problems with nonsmooth data; see, for 
example, nonsmooth weak maximum principles, in the spirit of (Clarke, 1983), derived in (Pales and Zeidan, 1994; de Pinho and Ilchmann, 2002; de Pinho, 2003) and strong versions of the nonsmooth maximum principle in (de Pinho et al., 2001) and (Devdariani and Ledyaev, 1996).

Roughly speaking, one may say that when the data of the problem under consideration is assumed to be continuous with respect to $t$ necessary conditions have previously been derived under several regularity assumptions the more general being the full rank assumption

$$
\operatorname{det} \Upsilon(t) \Upsilon(t)^{T} \neq 0 \text { for all } t \in[0,1]
$$

where

$$
\Upsilon(t)=\left[\begin{array}{c}
\nabla_{u} b(t, \bar{x}(t), \bar{u}(t)) \\
\nabla_{u} g^{\mathcal{I}_{a}(t)}(t, \bar{x}(t), \bar{u}(t))
\end{array}\right] .
$$

On the other hand, when the data is assumed to be merely measurably with respect to $t$ (a standard assumption for nonsmooth problems), the aforementioned full rank condition (4) on matrix $\Upsilon(t)$ is replaced by the uniformly full rank condition

$$
\operatorname{det} \Upsilon(t) \Upsilon(t)^{T} \geq K \quad \text { ae }
$$

where $K$ is some positive constant. See (de Pinho, 2003 ) in this respect.

Under mild conditions on the data, smooth weak maximum principles for $(P)$ under the previous full rank conditions ((4) or (6)) assert the existence of an absolutely continuous function $p$, integrable functions $q, r$ and a scalar $\lambda>0$ such that
a. $\lambda+\|p\|_{\infty}>0$,
b. $\dot{p}(t)=H_{x}(t, \bar{x}(t), \bar{u}(t), p(t), q(t), r(t)) \quad$ ae,
c. $0=H_{u}(t, \bar{x}(t), \bar{u}(t), p(t), q(t), r(t))$ ae,
d. $r(t) \cdot g(t, \bar{x}(t), \bar{u}(t))=0, \quad r(t) \leq 0 \quad$ ae,
e. $(p(0),-p(1)) \in N_{C}(\bar{x}(0), \bar{x}(1))+\lambda \nabla l(\bar{x}(0), \bar{x}(1))$,

f. There exists a $K_{1} \in L^{1}$ such that $|(q(t), r(t))| \leq K_{1}(t)|p(t)|$ ae.

Here $N_{C}(\bar{x}(0), \bar{x}(1))$ denotes the limiting normal cone of the set $C$ at $(\bar{x}(0), \bar{x}(1)$ ) (see for example (Vinter, 2000)).

It is of foremost importance to observe that there is no measures in the above conditions.

Problem $(P)$ can still be "regular" even in situations where full rank conditions on $\Upsilon(t)$ are not satisfied. Indeed, the set of necessary conditions $\mathrm{a}-\mathrm{f}$ for smooth problems or those given by (de Pinho, 2003, Theorem 3.1), for nonsmooth problems, still hold when full rank conditions are replaced by Mangasarian-Fromowitz constraints qualifications, also known as positively linear independence conditions on the mixed constraints (here we refer the reader to (Dmitruk, 1993)and (de Pinho, 2005)). To simplify the exposition we state here only a "uniform" version of such constraints qualifications.

HMF (de Pinho, 2005) There exist constants $K_{1}>0, K_{2}>0$ and integrable functions

$$
h, a:[0,1] \rightarrow R^{k}
$$

such that, for almost every $t \in[0,1],|h(t)|=1$ and

i. $a_{i}(t) \geq K_{2}$ ae for all $i \in \mathcal{I}_{a}(t)$,

ii. $\nabla_{u} \bar{g}(t) \cdot h(t)=a(t)$ ae,

iii. $\nabla_{u} \bar{b}(t) \cdot h(t)=0$ ae,

iv. $\operatorname{det} \nabla_{u} \bar{b}(t) \nabla_{u} \bar{b}(t)^{T} \geq K_{1}$, ae.

It is a simple matter to see that the uniform full rank (6) implies HMF whereas the opposite implication is not in general true.

In this paper we concentrate on necessary conditions for mixed constrained problems in the form of $(P)$ when Mangasarian-Fromowitz constraints qualifications are not satisfied. Those are problems we call nonregular. Since derivation of necessary conditions for nonregular problems remains a largely unexplored subject (see (Dmitruk, 1993)), our aim is to give some insight on the necessary conditions for such problems.

We do not consider general nonregular problems. Rather we focus attention on a simple class of mixed constrained problems for which matrix $\Upsilon(t)$ looses rank. The problem of interest is a particular case of the following linear problem

$$
\left\{\begin{aligned}
\text { Minimize } l(x(0), x(1)) & \\
\text { subject to } & \\
\dot{x}(t) & =A(t) x(t)+B(t) u(t) \quad \text { a.e. } \\
0 & \geq D x(t)+E u(t) \\
(x(0), x(1)) & \in C
\end{aligned}\right.
$$

where $l$ and $C$ are as defined before, $A$ and $B$ are matrix valued functions where $A(t) \in \mathcal{M}_{n \times n}$ and $B(t) \in \mathcal{M}_{n \times k}, D$ and $E$ are constant matrices such that $D \in \mathcal{M}_{m \times k}$ and $E \in \mathcal{M}_{m \times k}$, with $k \geq m$. Here $\mathcal{M}_{p \times q}$ denotes the set of real $p \times q$ matrices.

We show that when some hypotheses on matrix $E$ are satisfied problems in the form of $(L)$ are nonregular and additionally, they can be reduced to problems with pure state constraints. Such reduction permit the derivation necessary conditions for $(L)$. Notably, such necessary conditions differ from those given by $\mathrm{a}-\mathrm{f}$ above since they involve measures. 
Essential in the forthcoming development is the generalization of a weak nonsmooth maximum principle recently established in (de Pinho et al., 2005) for optimal control problems with pure state constraints.

Although our main result in this paper, Theorem 3.1 below, can be easily generalized to cover more general problems (for example, problems with nonlinear dynamics of the form $\dot{x}(t)=$ $f(t, x(t), u(t)))$ we opt to treat here the simpler problem $(L)$. Indeed, the special structure of $(L)$ allows us, at the same time, to simplify the exposition and to highlight the special features of nonregular problems.

This paper is organized in the following way. In the next section we give some essential definitions and, for completeness, we state a generalization of a weak nonsmooth maximum principle obtained in (de Pinho et al., 2005) to cover problems where the pure state constraints can be vector-valued. In section 3, we state and discuss our main result, the proof of which is presented in the last section.

\section{PRELIMINARIES}

Here and throughout, $B$ represents the closed unit ball centered at the origin and $|\cdot|$ the Euclidean norm or the induced matrix norm on $\mathbb{R}^{m \times k}$. The Euclidean distance function with respect to $A \subset$ $\mathbb{R}^{k}$ is

$d_{A}: \mathbb{R}^{k} \rightarrow \mathbb{R}, \quad y \mapsto d_{A}(y)=\inf \{|y-x|: x \in A\}$.

Here the linear space $W^{1,1}\left([0,1] ; \mathbb{R}^{p}\right)$ denotes the space of absolutely continuous functions, $L^{1}\left([0,1] ; \mathbb{R}^{p}\right)$ the space of integrable functions and $L^{\infty}\left([0,1] ; \mathbb{R}^{p}\right)$ the space of essentially bounded functions from $[0,1]$ to $\mathbb{R}^{p}$.

Crucial to all the analysis is the following result concerning the singular value decomposition of a matrix:

Theorem 2.1. (Horn and Jonhson, 1985, Theorem 7.3.5) If $E \in \mathcal{M}_{m \times k}$ and has rank $m_{e}\left(m_{e} \leq\right.$ $\min \{m, k\})$, then there exist $V \in \mathcal{M}_{m \times m}, W^{T} \in$ $\mathcal{M}_{k \times k}, \Sigma \in \mathcal{M}_{m \times k}$ and $\Lambda \in \mathcal{M}_{m_{e} \times m_{e}}$ such that

$$
E=V \Sigma W^{T},
$$

where $V$ and $W$ are unitary,

$$
\Sigma=\left[\begin{array}{cc}
\Lambda & 0 \\
0 & 0
\end{array}\right] \quad \text { and } \quad \Lambda=\operatorname{diag}\left\{\sigma_{i i}\right\}_{i=1}^{m_{e}}
$$

with $\sigma_{i i}>0$.

Recall that if a matrix $U$ is unitary, then $U^{T} U=I$ and $U^{-1}=U^{T}$.

Consider now the following optimal control problem with vector valued pure state constraints:
$(S)$

$$
\left\{\begin{aligned}
\text { Minimize } l(x(0), x(1)) & \\
\text { subject to } & \\
\dot{x}(t) & =f(t, x(t), u(t)) \quad \text { a.e. } \\
0 & \geq h(t, x(t)) \quad \text { for all } t \\
(x(0), x(1)) & \in C
\end{aligned}\right.
$$

where $l$, fand $C$ are as defined before and $h$ : $[0,1] \times \mathbb{R}^{n} \rightarrow \mathbb{R}^{m_{h}}$, with $m_{h} \geq 1$.

Next we focus on $(S)$ with possibly nonsmooth data (a standard procedure since the publication of the seminal book (Clarke, 1983)). Thus various concepts from nonsmooth analysis will be mentioned. Among those are the limiting normal cone to a set $C, N_{C}$, the limiting subdifferential of $f, \partial f(x)$, and the Clarke subdifferential, co $\partial f$. The concepts of limiting normal cone and limiting subdifferential as well as the full calculus for these constructions in finite dimensions is described in, for example, (Rockafellar and Wets, 1998; Vinter, 2000; Mordukhovich, 2006).

In the case that the function $f$ is Lipschitz continuous near $x$, the convex hull of the limiting subdifferential, co $\partial f(x)$, coincides with the Clarke subdifferential, which may be defined directly (see (Clarke, 1983)).

For $(P)$ or $(S)$ a process is a pair $(x, u)$ comprising a function $x \in W^{1,1}\left([0,1] ; \mathbb{R}^{n}\right)$ and a measurable function $u:[0,1] \rightarrow \mathbb{R}^{k}$ satisfying the constraints. Take a process $(\bar{x}(t), \bar{u}(t))$ to $(S)$ or $(P)$ and a parameter $\varepsilon>0$. Define the set

$T_{\varepsilon}(t)=\{(x, u): x \in \bar{x}(t)+\varepsilon B, u \in \bar{u}(t)+\varepsilon B\}$.

Let $\bar{f}(t), \bar{b}(t), \bar{g}(t)$ etc, denote the corresponding function evaluated at $(t, \bar{x}(t), \bar{u}(t))$.

A process $(\bar{x}, \bar{u})$ is a weak minimizer to $(P)$ or $(S)$ if there exists some $\varepsilon>0$, such that it minimizes the cost over all processes $(x, u)$ which satisfy

$$
(x(t), u(t)) \in T_{\varepsilon}(t) \quad \text { a.e. }
$$

We now focus on $(S)$. The following hypotheses, which make reference to a parameter $\varepsilon>0$, are imposed:

H1. The function $t \rightarrow f(t, x, u)$ is Lebesgue measurable for each pair $(x, u)$ and there exists a function $K_{f}$ in $L^{1}([0,1] ; \mathbb{R})$ such that

$$
\begin{aligned}
& \quad\left|f(t, x, u)-f\left(t, x^{\prime}, u^{\prime}\right)\right| \leq K(t)\left|(x, u)-\left(x^{\prime}, u^{\prime}\right)\right| \\
& \text { for }(x, u),\left(x^{\prime}, u^{\prime}\right) \in T_{\varepsilon}(t) \text { ae. }
\end{aligned}
$$

H2. The endpoint constraint set $C$ is closed and $l$ is locally Lipschitz in a neighborhood of $(\bar{x}(0), \bar{x}(1))$.

H3. For $x \in \bar{x}(t)+\varepsilon B$ the function $t \rightarrow h(t, x)$ is continuous and there exists a scalar $K_{h}>0$ such that $x \rightarrow h(t, x)$ is Lipschitz of rank $K_{h}$ for all $t \in[0,1]$. 
For $(S)$ define the function

$$
H_{S}(t, x, p, u)=p \cdot f(t, x, u) .
$$

The following theorem is a straightforward generalization of Unmaximized Hamiltonian type conditions for optimal problems with pure state constraints, proved in (de Pinho et al., 2005). The proof consists on considering a scalar functional

$$
h^{+}(t, x)=\max \left\{h_{j}(t, x): j \in\left\{1, \ldots, m_{h}\right\}\right\}
$$

following the approach in (Vinter, 2000).

Theorem 2.2. Let $(\bar{x}, \bar{u})$ be a weak minimizer to problem $(S)$. Assume that $\mathrm{H} 1-\mathrm{H} 3$ are satisfied for some parameter $\varepsilon$. Then there exists an absolutely continuous function $p:[0,1] \mapsto \mathbb{R}^{n}$, integrable function $\gamma_{j}:[0,1] \rightarrow \mathbb{R}^{n}$, for $j=1, \ldots, m_{h}$, nonnegative Radon measures $\mu_{j} \in C^{*}([0,1], \mathbb{R})$, $j=1, \ldots, m_{h}$, and a scalar $\lambda \geq 0$ such that

$$
\begin{aligned}
& \text { i. } \sum_{j=1}^{m_{h}} \mu_{j}\{[0,1]\}+\|p\|_{\infty}+\lambda>0, \\
& \text { ii. }(-\dot{p}(t), 0) \in \operatorname{co} \partial_{x, u} H(t, \bar{x}(t), \pi(t), \bar{u}(t)) \text { a.e. }
\end{aligned}
$$

iii. $(p(0),-\pi(1)) \in$

$$
N_{C}(\bar{x}(0), \bar{x}(1))+\lambda \partial l(\bar{x}(0), \bar{x}(1)),
$$

where

$$
\operatorname{supp}\left\{\mu_{j}\right\} \subset\left\{t \in[0,1]: h_{j}(t, \bar{x}(t))=0\right\},
$$

$\pi$ is defined as

$$
\pi(t)=\left\{\begin{array}{l}
p(t)+\int_{[0, t)} \sum_{j=1}^{m_{h}} \gamma_{i}(\tau) \mu_{j}(d \tau), t \in[0,1) \\
p(t)+\int_{[0,1]} \sum_{j=1}^{m_{h}} \gamma_{i}(\tau) \mu_{j}(d \tau), \quad t=1 .
\end{array}\right.
$$

and

$$
\gamma_{j}(t) \in \partial_{x}^{>} h_{j}(t, \bar{x}(t)) \mu_{j} \text {-a.e. }
$$

with

$$
\begin{aligned}
& \partial_{x}^{>} h_{j}(t, x)=\operatorname{co}\left\{\xi: \exists\left(t_{i}, x_{i}\right) \stackrel{h}{\rightarrow}(t, x):\right. \\
& \left.h_{j}\left(t_{i}, x_{i}\right)>0 \quad \forall i, \nabla_{x} h_{j}\left(t_{i}, x_{i}\right) \rightarrow \xi\right\} .
\end{aligned}
$$

\section{MAIN RESULT}

We shall invoke the following set of hypotheses on $(L)$ :

AH1. For almost every $t \in[0,1]$ we have

$$
\mathcal{I}_{a}(t)=\{1, \ldots, m\} \text {. }
$$

AH2. The matrix $E$ has rank $m_{e}$ where $1 \geq$ $m_{e}<m$. Writing $E=\left[\begin{array}{c}E_{e} \\ E_{s}\end{array}\right]$, then $E_{e} \in \mathcal{M}_{m_{e} \times k}$ is of full rank and there exists a matrix $Q \in$ $\mathcal{M}_{\left(m-m_{e}\right) \times m_{e}}$ such that $E_{s}=-Q E_{e}$.
AH3. The matrix valued function $t \rightarrow(A(t), B(t))$ is integrable.

Observe that in AH1 we assume that all the constraints are active. It follows that $E=E^{\mathcal{I}_{a}(t)}$. Hypothesis AH2 asserts that $E$ is not of full rank. Since we also assume the existence of a matrix $Q$ such that $E_{s}=-Q E_{e}$ it follows that HMF is not satisfied. Thus, when the data of $(L)$ satisfies $\mathrm{AH} 1-\mathrm{AH} 2,(L)$ is a nonregular problem. In this respect it is important to observe that the rank of $E$ is assumed to be greater or equal than 1 .

Taking into account the partition of matrix $E$ in AH2, write $D=\left[\begin{array}{c}D_{e} \\ D_{s}\end{array}\right]$ and $B(t)=\left[B_{e}(t) \mid B_{s}(t)\right]$, where $D_{e} \in \mathcal{M}_{m_{e} \times n}, B_{e}(t) \in \mathcal{M}_{n \times m_{e}}, D_{s} \in$ $\mathcal{M}_{\left(m-m_{e}\right) \times n}$ and $B_{s}(t) \in \mathcal{M}_{n \times\left(k-m_{e}\right)}$.

In what follows

$$
\hat{D}=Q D_{e}+D_{s} .
$$

Theorem 3.1. Let $(\bar{x}, \bar{u})$ be a weak minimizer to $(L)$. If hypotheses $\mathrm{H} 2, \mathrm{AH} 1, \mathrm{AH} 2$ and $\mathrm{AH} 3$ are satisfied, then there exist an absolutely continuous function $p:[0,1] \mapsto \mathbb{R}^{n}$, integrable function $r:[0,1] \rightarrow \mathbb{R}^{m_{e}}$, nonnegative Radon measures $\mu_{j} \in C^{*}([0,1], \mathbb{R})$, with $j=1, \ldots, m-m_{e}$, and a scalar $\lambda \geq 0$ such that

$$
\begin{aligned}
& \text { i. } \quad \sum_{j=1}^{m-m_{e}} \mu_{j}\{[0,1]\}+\|p\|_{\infty}+\lambda>0, \\
& \text { ii. } \quad-\dot{p}(t)=\pi(t) \cdot A(t)+r(t) \cdot D_{e} \text { a.e. } \\
& \text { iii. } \quad 0=\pi(t) \cdot B(t)+r(t) \cdot E_{e} \text { a.e. } \\
& \text { iv. } \quad(p(0),-\pi(1)) \in \\
& \quad \lambda \partial l(\bar{x}(0), \bar{x}(1))+N_{C}(\bar{x}(0), \bar{x}(1)),
\end{aligned}
$$

where

$$
\operatorname{supp}\left\{\mu_{j}\right\} \subset\left\{t \in[0,1]: \hat{d}_{j} \bar{x}(t)=0\right\} ，
$$

and $\pi$ is defined as

$$
\pi(t)=\left\{\begin{array}{l}
p(t)+\int_{[0, t)} \sum_{j=1}^{m-m_{e}} \hat{d}_{j} \mu_{j}(d \tau), t \in[0,1) \\
p(t)+\int_{[0,1]} \sum_{j=1}^{m-m_{e}} \hat{d}_{j} \mu_{j}(d \tau), \quad t=1 .
\end{array}\right.
$$

with $\hat{d}_{j}$ being the j-th row of $\hat{D}$.

A remarkable difference between the conclusions of Theorem 3.1 and the necessary conditions given by a-f above is that $r$, the multiplier associated with mixed constraints, is now defined by (iii) in terms of the bounded variation function $\pi$ and not in terms of the absolutely continuous function $p$. 
Although AH1-AH2 are strong assumptions on the data of $(L)$ they nevertheless are of interest since they permit the illustration of some special features of nonregular problems in a simple and straightforward way. We emphasize the fact that necessary conditions analogous as those given by the above theorem can be derived when AH1-AH2 are replaced by weaker assumptions. Theorem 3.1 holds when, for example, when the constraints are active in subinterval of $[0,1]$ and not in the whole interval. However the proof of this Theorem would be much more demanding. Also, and as stated in the Introduction, Theorem 3.1 can easily be generalized to cover problems with nonsmooth dynamics of the form $\dot{x}(t)=f(t, x(t), u(t))$.

Nondegeneracy of the necessary conditions given by Theorem 3.1 shall be treated in future work.

\section{PROOF OF MAIN RESULT}

Now we prove Theorem 3.1. We start by proving that $(L)$ can be associated with a problem in the form of $(S)$. In this respect $\mathrm{AH} 2$ is crucial.

Consider the full rank matrix $E_{e}$. Theorem 2.1 asserts the existence of unitary matrices $V_{e}$ and $W_{e}$, of real positive numbers $\sigma_{i i}, i \in\left\{1, \ldots, m_{e}\right\}$ such that

$$
E_{e}=V_{e}\left[\Lambda_{e} \mid 0\right] W_{e}^{T},
$$

where

$$
\Lambda=\operatorname{diag}\left\{\sigma_{i i}\right\}_{i=1}^{m_{e}} .
$$

Take any vector $u \in \mathbb{R}^{k}$ and define $v=W_{e}^{T} u \in$ $R^{k}$. Write

$$
v=\left[\begin{array}{l}
v_{e} \\
v_{s}
\end{array}\right]
$$

where $v_{s} \in \mathbb{R}^{k-m_{e}}$. Likewise write $\bar{v}(t)=W_{e}^{T} \bar{u}(t)$.

Set also

$$
\begin{aligned}
{\left[\hat{B}_{e}(t) \mid \hat{B}_{s}(t)\right] } & =B(t) W_{e} \\
\hat{A}(t) & =A(t)-\hat{B}_{e}(t) \Lambda_{e}^{-1} V_{e}^{T} D_{e} \\
\hat{D} & =Q D_{e}+D_{s} .
\end{aligned}
$$

Proposition 4.1. If $(\bar{x}, \bar{u})$ is a weak minimizer to $(L)$, then $\left(\bar{x}, \bar{v}_{s}\right)$, is a weak minimizer for

$$
\left(L_{\text {aux }}\right) \quad\left\{\begin{array}{l}
\text { Minimize } l(x(1)) \\
\text { subject to } \\
\dot{x}(t)=\hat{A}(t) x(t)+\hat{B}_{s}(t) v_{s}(t) \quad \text { ae } \\
0 \geq \hat{D} x(t) \\
(x(0), x(1)) \in C
\end{array}\right.
$$

Proof. It is a simple matter to see that if $(\bar{x}, \bar{u})$ solves $(L)$, then $\left(\bar{x}, \bar{v}_{e}\right)$ is admissible to $\left(L_{a u x}\right)$. Indeed, we have

$$
D \bar{x}(t)+E \bar{u}(t)=0 \Longleftrightarrow\left\{\begin{array}{l}
D_{e} \bar{x}(t)+E_{e} \bar{u}(t)=0 \\
D_{s} \bar{x}(t)+E_{s} \bar{u}(t)=0
\end{array}\right.
$$

for almost every $t \in[0,1]$. Premultiply the first equation of the above system by $Q$. This, together with $\mathrm{AH} 2$, yields

$$
Q D_{e} \bar{x}(t)=-Q E_{e} \bar{u}(t)=E_{s}(t) \bar{u}(t) .
$$

Thus

$$
\begin{cases}D_{e} \bar{x}(t)+E_{e} \bar{u}(t)=0 & \text { a.e. } \\ \hat{D} \bar{x}(t)=0 & \text { a.e. }\end{cases}
$$

But $\hat{D}$ is a constant matrix and $\bar{x}$ is absolutely continuous. So the equality $\hat{D} \bar{x}(t)=0$ holds for all $t \in[0,1]$.

Now consider $\bar{v}(t)=W_{e}^{T} \bar{u}(t)$. Observe that

$$
\begin{gathered}
D_{e} \bar{x}(t)+E_{e} \bar{u}(t) \\
=D_{e} \bar{x}(t)+V_{e}\left[\Lambda_{e} \mid 0\right] W_{e}^{T} \bar{u}(t) \\
=D_{e} \bar{x}(t)+V_{e}\left[\Lambda_{e} \mid 0\right] \bar{v}(t) \\
=0
\end{gathered}
$$

Premultiply this last equation by $V_{e}^{T}$ we deduce that

$$
V_{e}^{T} D_{e} \bar{x}(t)+\Lambda_{e} \bar{v}_{e}(t)=0 \quad \text { a.e. }
$$

It follows that

$$
\bar{v}_{e}(t)=-\Lambda_{e}^{-1} V_{e}^{T} D_{e} \bar{x}(t) .
$$

We also have

$$
\begin{gathered}
A(t) \bar{x}(t)+B(t) \bar{u}(t) \\
=A(t) \bar{x}(t)+B(t) W_{e} \bar{v}(t) \\
=A(t) \bar{x}(t)+\hat{B}_{e}(t) \bar{v}_{e}(t)+\hat{B}_{s}(t) \bar{v}_{s}(t) \\
=\left(A(t)-\hat{B}_{e}(t) \Lambda_{e}^{-1} V_{e}^{T} D_{e}\right) \bar{x}(t)+\hat{B}_{s}(t) \bar{v}_{s}(t) .
\end{gathered}
$$

We conclude from the above that

$$
\dot{\bar{x}}(t)=\hat{A}(t) \bar{x}(t)+\hat{B}_{s}(t) \bar{v}_{s}(t) .
$$

It follows that $\left(\bar{x}, \bar{v}_{s}\right)$ is admissible to $\left(L_{a u x}\right)$.

Suppose now that $\left(\tilde{x}, \tilde{v}_{s}\right)$ is an admissible solution to $\left(L_{a u x}\right)$ with lesser cost. Set

$$
\tilde{v}_{e}(t)=-\Lambda_{e}^{-1} V_{e}^{T} D_{e} \tilde{x}(t), \quad \tilde{v}(t)=\left[\begin{array}{c}
\tilde{v}_{e}(t) \\
\tilde{v}_{s}(t)
\end{array}\right]
$$

and define

$$
\tilde{u}(t)=W_{e} \tilde{v}(t) .
$$

As it can easily be verified we have

$$
\dot{\tilde{x}}(t)=A(t) \tilde{x}(t)+B(t) \tilde{u}(t) \quad \text { a.e. }
$$

Also

$$
\begin{gathered}
V_{e}^{T} D_{e} \tilde{x}(t)+\Lambda_{e} \tilde{v}_{e}(t) \\
=V_{e}^{T} D_{e} \tilde{x}(t)+\left[\Lambda_{e} \mid 0\right] \tilde{v}(t) \\
=V_{e}^{T} D_{e} \tilde{x}(t)+\left[\Lambda_{e} \mid 0\right] W_{e}^{T} \tilde{u}(t) \\
=0 .
\end{gathered}
$$

Premultiplying this equation by $V_{e}$ we get

$$
D_{e} \tilde{x}(t)+E_{e} \tilde{u}(t)=0 .
$$

Again premultiply this last equation by $Q$. Appealing to $\mathrm{AH} 2$ we deduce that

$$
Q D_{e} \tilde{x}(t)=E_{s} \tilde{u}(t) \text {. }
$$


Taking into account the definition of $\hat{D}$ we now deduce that

$0 \geq \hat{D} \tilde{x}(t)=\left(Q D_{e}+D_{s}\right) \tilde{x}(t)=D_{s} \tilde{x}(t)+E_{s} \tilde{u}(t)$.

We conclude that

$$
\begin{aligned}
& D_{e} \tilde{x}(t)+E_{e} \tilde{u}(t)=0 \\
& D_{s} \tilde{x}(t)+E_{s} \tilde{u}(t) \leq 0
\end{aligned}
$$

that is, $(\tilde{x}, \tilde{u})$ is an admissible solution to $(L)$ with lesser cost, contradicting the optimality of $(\bar{x}, \bar{u})$.

It is an easy task to verify that $\left(L_{a u x}\right)$ is an optimal control with pure state constraints satisfying the conditions under which Theorem 2.2 holds. Application of Theorem 2.2 asserts the existence of an absolutely continuous function $p:[0,1] \mapsto \mathbb{R}^{n}$, nonnegative Radon measures $\mu_{j} \in C^{*}([0,1], \mathbb{R})$, with $j=1, \ldots, m-m_{e}$, and a scalar $\lambda \geq 0$ such that (i) and (7) of the Theorem holds with $\pi$ as defined in (8). Moreover

$$
\begin{aligned}
-\dot{p}(t) & =\pi(t) \cdot \hat{A}(t) \\
& =\pi(t) \cdot A(t)-\pi(t) \cdot \hat{B}_{e}(t) \Lambda_{e}^{-1} V_{e}^{T} D_{e} \\
0 & =\hat{B}_{s}(t) \cdot \pi(t)
\end{aligned}
$$

Define the vector valued function $r:[0,1] \rightarrow \mathbb{R}^{m_{e}}$ by

$$
\left[\begin{array}{c}
r(t) \\
0
\end{array}\right]=-\left[\begin{array}{cc}
V_{e} & 0 \\
0 & I
\end{array}\right]\left[\begin{array}{cc}
\Lambda_{e}^{-1} & 0 \\
0 & I
\end{array}\right] W_{e}^{T} B^{T}(t) \pi(t)
$$

where $\left[\begin{array}{c}r(t) \\ 0\end{array}\right] \in \mathbb{R}^{k}$.

Then (ii) and (iii) follows. The proof is complete.

\section{REFERENCES}

Arutyunov, A. V. (2000). Optimality Conditions: Abnormal and Degenerate Problems. Kluwer Academic Publishers, Dordrecht.

Clarke, F. H. (1983). Optimization and Nonsmooth Analysis. Wiley, New York.

de Pinho, M.d.R. (2003). Mixed constrained control problems. Journal of Mathematical Analysis and Applications 278, 293-307.

de Pinho, M.d.R (2005). Necessary conditions under mangasarian-fromowitz type assumptions for mixed constrained control problems. In: Proceedings of CDC-ECC'05, Seville, Spain. pp. 266-270.

de Pinho, M.d.R. and A. Ilchmann (2002). Weak maximum principle for optimal control problems with mixed constraints. Nonlinear Analysis, Theory and Applications 48, 1179-1196. de Pinho, M.d.R., M. M. A. Ferreira and F. A. C. C. Fontes (2005). Unmaximized inclusion necessary conditions for nonconvex constrained optimal control problems. ESAIM, Control Optim. Cal. Var. de Pinho, M.d.R., R.B. Vinter and H. Zheng (2001). A maximum principle for optimal control problems with mixed constraints. IMA Journal of Math. Control and Information 18, 189-205.

Devdariani, E.N. and Y. S. Ledyaev (1996). Maximum principle for implicit control systems. Appl. Math. Optim. 40, 79-103.

Dmitruk, A. V. (1993). Maximum principle for the general optimal control problem with phase and regular mixed constraints. Computation Mathematics and Moddeling, CMMOEA 4, 307-426.

Hestenes, M. R. (1966). Calculus of Variations and Optimal Control. Wiley, New York.

Horn, R. A. and C. R. Jonhson (1985). Matrix Analysis. Cambridge Press.

Milyutin, A. A. and N. P. Osmolovskii (1998). Calculus of Variations and Optimal Control Theory. American Mathematical Society. Providence, Rhode Island.

Mordukhovich, B. S. (2006). Variatonal Analysis and Generalized Dofferention I. Springer.

Neustadt, L. W. (1976). Optimization, A Theory of Necessary Conditions. Princeton University Press. New Jersey.

Pales, Z. and V. Zeidan (1994). First and second order necessary conditions for control problems with constraints. Transc. American Mathematical Society 346, 421-453.

Rockafellar, R. T. and B. Wets (1998). Variational Analysis. Springer, Berlin.

Schwarzkopf, A. B. (1976). Relaxed control problems with equality state constraints. SIAM J. Optim. Theory and Applic. 19, 455-468.

Steffani, G. and P.L. Zezza (1996). Optimality conditions for constrained control problems. SIAM Journal of Control and Optimization 34, 635-659.

Vinter, R. B. (2000). Optimal Control. Birkhouser. 